\title{
TERT structural rearrangements in metastatic pheochromocytomas
}

\author{
Trisha Dwight, ${ }^{1,2}$ Aidan Flynn3,4, Kaushalya Amarasinghe5, Diana E Benn1,2, Richard Lupat5, Jason Li5, \\ Daniel L Cameron5,6,7, Annette Hogg5, Shiva Balachander5, Ida L M Candiloro8,9, Stephen Q Wong5, \\ Bruce G Robinson1,2, Anthony T Papenfuss5,6,7,10,11, Anthony J Gill2,12, Alexander Dobrovic8,9,13, Rodney J Hicks5,10, \\ Roderick J Clifton-Bligh',2 and Richard W Tothill5,9,10
}

\author{
${ }^{1}$ Cancer Genetics, Kolling Institute, Royal North Shore Hospital, Sydney, New South Wales, Australia \\ ${ }^{2}$ The University of Sydney, Sydney, New South Wales, Australia \\ ${ }^{3}$ The Finsen Laboratory, Rigshospitalet, Faculty of Health Sciences, University of Copenhagen, Copenhagen N, Denmark \\ 4Biotech Research and Innovation Centre (BRIC), University of Copenhagen, Copenhagen N, Denmark \\ 5The Peter MacCallum Cancer Centre, East Melbourne, Victoria, Australia \\ ${ }^{6}$ Bioinformatics Division, The Walter and Eliza Hall Institute of Medical Research, Parkville, Victoria, Australia \\ ${ }^{7}$ Department of Medical Biology, University of Melbourne, Melbourne, Victoria, Australia \\ 8 Olivia Newton-John Cancer Research Institute, Heidelberg, Victoria, Australia \\ ${ }^{9}$ The Department of Pathology, University of Melbourne, Parkville, Victoria, Australia \\ ${ }^{10}$ The Sir Peter MacCallum Department of Oncology, The University of Melbourne, Parkville, Victoria, Australia \\ ${ }^{11}$ The Department of Mathematics and Statistics, University of Melbourne, Parkville, Victoria, Australia \\ ${ }^{12}$ Cancer Diagnosis and Pathology Group, Kolling Institute, Royal North Shore Hospital, Sydney, New South Wales, Australia \\ ${ }^{13} \mathrm{~S}$ chool of Cancer Medicine, La Trobe University, Bundoora, Victoria, Australia
}

Correspondence should be addressed to T Dwight or R Tothill: trisha.dwight@sydney.edu.au or rtothill@unimelb.edu.au

\begin{abstract}
Pheochromocytomas (PC) and paragangliomas (PGL) are endocrine tumors for which the genetic and clinicopathological features of metastatic progression remain incompletely understood. As a result, the risk of metastasis from a primary tumor cannot be predicted. Early diagnosis of individuals at high risk of developing metastases is clinically important and the identification of new biomarkers that are predictive of metastatic potential is of high value. Activation of TERT has been associated with a number of malignant tumors, including PC/PGL. However, the mechanism of TERT activation in the majority of PC/PGL remains unclear. As TERT promoter mutations occur rarely in PC/PGL, we hypothesized that other mechanisms - such as structural variations - may underlie TERT activation in these tumors. From 35 PC and four PGL, we identified three primary PCs that developed metastases with elevated TERT expression, each of which lacked TERT promoter mutations and promoter DNA methylation. Using whole genome sequencing, we identified somatic structural alterations proximal to the TERT locus in two of these tumors. In both tumors, the genomic rearrangements led to the positioning of super-enhancers proximal to the TERT promoter, that are likely responsible for the activation of the normally tightly repressed TERT expression in chromaffin cells
\end{abstract}

\author{
Key Words \\ - TERT \\ - pheochromocytoma \\ - metastatic \\ - whole genome sequencing \\ - rearrangements
}




\section{Introduction}

Pheochromocytomas (PC) and paragangliomas (PGLs) are neuroendocrine tumors derived from neural crest cells of the sympathetic and parasympathetic nervous system - PCs arising in the adrenal medulla and PGLs in paraganglia outside the adrenal medulla. Although rare, they often occur as part of hereditary endocrine tumor syndromes and their study has been richly rewarding for identifying genetic events that drive tumorigenesis (Castro-Vega et al. 2015a). Metastatic disease occurs in approximately $10 \%$ of PC and up to $40 \%$ of PGL (CastroVega et al. 2015a); however, it is now accepted that all PC/PGL have some metastatic potential; therefore, the concept of 'benign' and 'malignant' is no longer applicable (Tischler et al. 2017). Despite germline mutations in succinate dehydrogenase subunit $B$ $(S D H B)$ and fumarate hydratase $(F H)$ being associated with metastatic disease (SDHB (Benn et al. 2015), FH (Castro-Vega et al. 2014)), genetic associations with most metastatic PC/PGL remain unknown. Identification of new genetic biomarkers of metastatic potential are of high priority, given there are no recommended molecular or histopathological tumor features that can be used to guide patient management (Tischler et al. 2017).

Activation of TERT as a result of promoter mutations (specifically, chr5:1295228C $>\mathrm{T}$ and chr5:1295250C $>\mathrm{T}$ ) were initially found in melanoma (Horn et al. 2013) and have subsequently been shown to be one of the most frequent somatic mutations in cancer (reviewed in Ramlee et al. 2016). Although approximately 25\% of all PC/PGLs overexpress TERT (Liu et al. 2014), promoter mutations are rare $(1.7 \%$ (Liu et al. 2014); 1.2\% (Papathomas et al. 2014)), suggestive of another mechanism of TERT activation. Recently, activation of TERT as a result of structural rearrangements, leading to the placement of enhancers proximal to the TERT promoter have been described in a number of cancer types (Davis et al. 2014, Peifer et al. 2015, Valentijn et al. 2015, Alaei-Mahabadi et al. 2016, Barthel et al. 2017). We therefore hypothesized that structural alterations involving the TERT promoter may also contribute to PC/PGL. We identified three metastatic PCs with elevated TERT expression and using whole genome sequencing (WGS) identified genomic rearrangements that led to super-enhancer positioning proximal to the TERT promoter in two tumors.

\section{Materials and methods}

\section{Patients and samples}

Informed and written patient consent was obtained for the collection and study of all samples, under approval of the Northern Sydney Local Health District (Protocol \#1103-107M) and Peter MacCallum Cancer Centre (Protocol \#10-119) Human Research Ethics Committees. The patient clinical data have been described previously (Flynn et al. 2015).

\section{RNA sequencing analysis}

RNA-seq data were available for 35 PCs and four PGLs described in a previous study (Flynn et al. 2015). RNAseq reads mapping to TERT were converted to gene feature counts then normalized for sequencing depth. Visualization of exon-level read depth and TERT spliceisoform usage was done using the Sashimi function in Integrated Genomics Viewer (Robinson et al. 2011) (version 2.3.68).

\section{Targeted amplicon sequencing for detection of TERT promoter mutations}

PCR primers were designed across base pair positions at canonical TERT promoter mutations, chr5:1295228C $>\mathrm{T}$ and chr5:1295250C $>\mathrm{T}$ and contained universal 5-prime sequences compatible with Fluidigm CS1 and CS2 primers (Fluidigm, San Francisco, CA, USA). Following PCR amplification of genomic DNA, PCR products were pooled and then subject to secondary PCR using indexed CS1 and CS2 primers (Fluidigm) before sequencing on a MiSeq platform $(2 \times 150$ bp) (Illumina, San Diego, CA, USA). Primer design and methods for read alignment and variant calling have been previously described (Wong et al. 2015).

\section{Methylation-sensitive high-resolution melting (MS-HRM)}

$100 \mathrm{ng}$ of tumor DNA was modified using the EZ DNA Methylation-Lightning kit (Zymo Research, Irvine, CA, USA) according to the manufacturer's instructions. Primer locations covered regions previously analyzed by Lee et al. (2017a) (first assay) and Castelo-Branco et al. (2013) (second assay), respectively. Primers for the first 
assay were 5'-CGGAGTTGGAAGGTGAAGGGGTA-3' and 5'-GCTAACNTCCCTACACCCTAAAAAC-3'. Primers for second assay were 5'-CGGGGAAGTGTTGTAGGGAGG-3' and 5'-GAAAACCCAAAACTACCTCCAAATCC-3'.

All PCR and MS-HRM reactions were performed on a Rotorgene 6000 (Corbett, Sydney, Australia). PCR reactions $(20 \mu \mathrm{L})$ contained $200 \mathrm{nmol} / \mathrm{L}$ of each primer, $200 \mu \mathrm{mol} / \mathrm{L}$ dNTPs (Fisher Biotec, Perth, Australia), $5 \mu \mathrm{mol} / \mathrm{L}$ SYTO 9 (Molecular Probes), $2.5 \mathrm{mmol} / \mathrm{L} \mathrm{MgCl}_{2}, 0.5 \mathrm{U}$ HotStarTaq DNA Polymerase in its supplied buffer $(1 \times)$ (Qiagen) and $0.5 \times$ Q-Solution (supplied with polymerase) in the supplied buffer $(1 \times)$ (Qiagen). Both MS-HRM-PCR conditions began with $95^{\circ} \mathrm{C}$ for $15 \mathrm{~min}$, and then 55 cycles of $95^{\circ} \mathrm{C}$ for $30 \mathrm{~s}, 63^{\circ} \mathrm{C}$ for $30 \mathrm{~s}$ and $72^{\circ} \mathrm{C}$ for $30 \mathrm{~s}$ for the first primer set; and $95^{\circ} \mathrm{C}$ for $10 \mathrm{~s}, 62^{\circ} \mathrm{C}$ for $20 \mathrm{~s}$ and $72^{\circ} \mathrm{C}$ for $20 \mathrm{~s}$ for the second primer set. HRM immediately followed the PCR with a hold at $97^{\circ} \mathrm{C}$ for $1 \mathrm{~min}, 72^{\circ} \mathrm{C}$ for 1 min then an analysis step from $65^{\circ} \mathrm{C}$ to $95^{\circ} \mathrm{C}$ rising $0.2^{\circ} \mathrm{C}$ per second and holding for $1 \mathrm{~s}$ at each increment.

\section{Whole genome sequencing (WGS)}

WGS was performed on paired DNA from fresh frozen tumor and peripheral blood. Sequencing libraries were prepared ( $1 \mu \mathrm{g}$ of DNA) according to the manufacturer's instructions (TruSeq Nano DNA HT Sample Prep Kit, Illumina) and sequenced on the Illumina HiSeq $X$ Ten platform (Kinghorn Centre for Clinical Genomics, Garvan Institute of Medical Research, Sydney, Australia). Sequencing reads were aligned to the UCSC hg19 reference genome using BWA-mem (Li 2013). Duplicate reads were marked using Picard (http://broadinstitute. github.io/picard). Mean sequence coverage in individual samples was 50-60× (Supplementary Table 2, see section on supplementary data given at the end of this article).

\section{Copy-number analysis}

Copy-number analysis was done using Fraction and AlleleSpecific Copy-Number Estimates from Tumor Sequencing (FACETS; version 0.5.6, segmentation c-value=500) (Shen $\&$ Seshan 2016)). Tumor purity estimates were based on subclonal model (Supplementary Table 2).

\section{Detection of genomic rearrangements}

Genomic rearrangements were identified using Genomic Rearrangement IDentification Software Suite (GRIDSS, version 0.11.5) (Cameron et al. 2017). Breakpoints were filtered out based on occurrence within ENCODE DAC blacklisted regions and presence within the germline. Reported breakpoints calls were supported by at least two read pairs, one split read and a breakend assembly contig at both respective breakpoint regions. They were also at least $2 \mathrm{~Kb}$ in size with less than 50 bp homology observed between the breakpoint ends and had GRIDSS confidence scores greater than 200 .

\section{Validation of genomic rearrangements by PCR and Sanger sequencing}

PCR was carried out in the tumor and matched normal genomic DNA using primers spanning the predicted breakpoints (chr5:1295558 and chr5:5601933 (V-PH-03T); chr5:1302401 and chr22:33960879 (V-PH-04T)), with the products subsequently analyzed by Sanger sequencing.

\section{Telomere length analysis}

Telomere length was assessed using TelSeq (Ding et al. 2014), a bioinformatic tool known to correlate with the standard experimental methods of qPCR and terminal restriction fragment (TRF)-length analysis (Lee et al. 2017a). Reads containing the telomeric repeat (TTAGGG) $\times 3$ or $($ CCCTAA) $\times 3$ were counted and normalized to the average genomic coverage (the average base coverage of each genome).

\section{Results}

\section{Overexpression of TERT in a subset of PC/PGL}

A focused re-analysis of RNA-seq data generated from our prior study (Flynn et al. 2015) showed detectable TERT mRNA expression in 20 of 39 (51\%) PC/PGL tumors (18 PC and two PGL), including five of six PCs that developed metastases (Fig. 1A, Supplementary Table 1). Markedly elevated TERT expression (4.66 (square root counts per million, SQRT-CPM), 5.07 SQRT-CPM) was observed in two metastatic PC (V-PH-01T, V-PH-04T, respectively) while a third metastatic PC (V-PH-03T) showed moderately elevated expression (0.98 SQRT-CPM). Analysis of splicemapped sequence reads corresponding to TERT exons 6-9 showed that full-length TERT, which is required for TERT enzymatic activity, could be detected in the moderateto-high expressing cases in addition to the common $\beta$-deletion ( $\beta-$ ) isoform that skips exons 7 and 8 (Fig. 1B).

Targeted amplicon sequencing did not detect canonical TERT promoter mutations in any of the 39 PC/PGL tumors analyzed. As TERT promoter methylation 


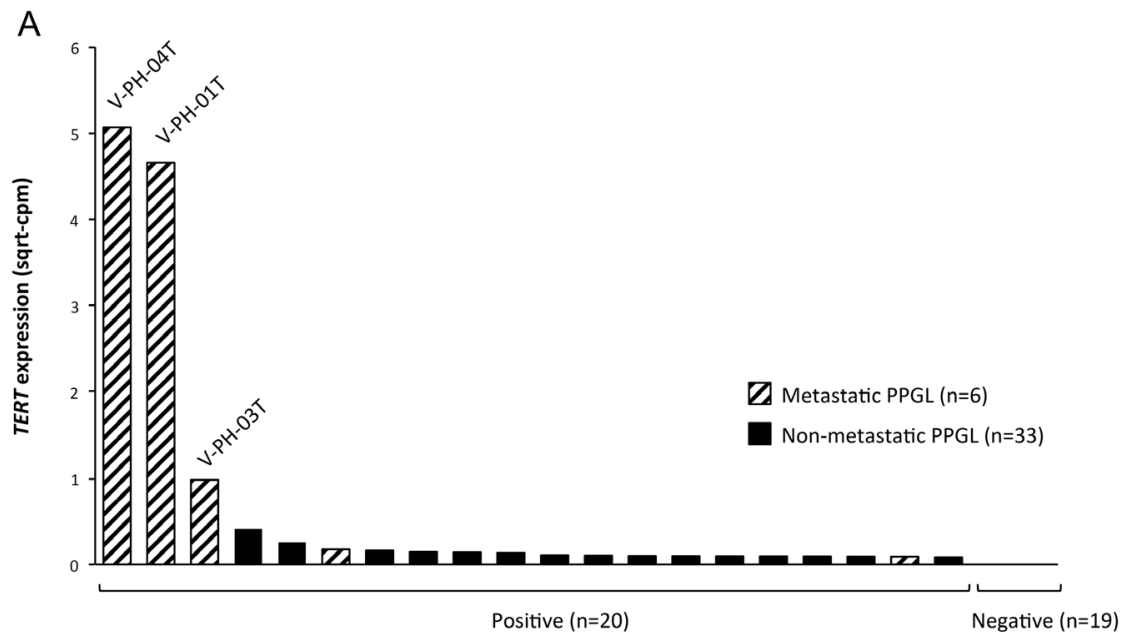

B

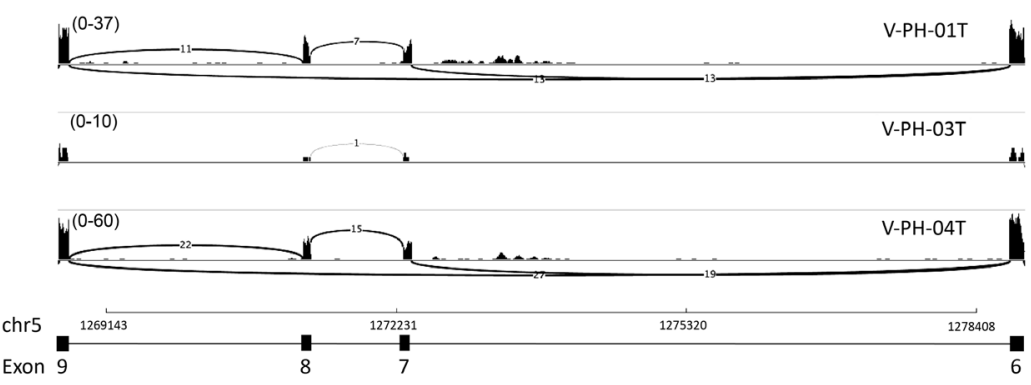

\section{Figure 1}

TERT expression in PC/PGL $(n=39)$ taken from RNA-seq data described in Flynn et al. (2015). (A) Square root of RNA-seq counts per million aligned to the TERT locus in 39 PC/PGL tumor samples. (B) Sashimi plot depicting mapped RNA-seq reads spanning TERT exons 6-9 (supporting read number shown in exon links) and read depth (maximum range shown in parenthesis). Exons 7-8 are spliced out in $\beta$-deletion ( $\beta-$ ) isoform.

has also shown to be positively correlated with TERT expression in other cancer types, such as pediatric brain cancers and neuroblastoma, we used high-resolution melting following DNA bisulfite conversion and PCR amplification (MS-HRM) to target two distinct regions of the promoter $\mathrm{CpG}$ island studied by other groups (Castelo-Branco et al. 2013, Lee et al. 2017b). The limit of detection using HRM-PCR is $\sim 1 \%$ depending on the degree of methylation heterogeneity at the CpG sites. We did not detect any DNA methylation at target $\mathrm{CpG}$ regions in the 39 tumors analyzed (Supplementary Fig. 1). We therefore excluded canonical TERT promoter mutations and DNA methylation as possible mechanisms of TERT dysregulation in our tumor cohort.

Whole genome sequencing, telomere length analysis and identification of TERT promoter rearrangements in PC

We performed WGS on tumor and matching blood for the moderate-to-high TERT-expressing cases (V-PH-01T, $\mathrm{V}-\mathrm{PH}-03 \mathrm{~T}$ and V-PH-04T). An in silico analysis of telomere length utilizing the WGS data indicated that telomeres were shorter in tumors V-PH-01T and V-PH-04T compared to their matched normal blood controls, whereas
V-PH-03T had an approximately equal telomere length to the matched blood (Supplementary Table 3). Our observation of reduced telomere length in tumor compared to matched blood in two of our TERT-overexpressing tumors is consistent with a recent observation made across 31 tumor types (Barthel et al. 2017). Importantly, we note that case V-PH-03T had a relatively low tumor purity assessed by FACETS ( 30\%), and this may have reduced the accuracy of telomere length estimation and level of detected TERT expression in this case.

Structural variant analysis of the WGS data indicated that V-PH-03T and V-PH-04T had complex patterns of somatic rearrangement including regions of localized genomic instability (V-PH-03T: chr5p, chr7q; and V-PH04T: chr3p, chr11p, chr22q; Fig. 2A). In contrast, V-PH01T had no detectable structural rearrangements with the presence of whole chromosome or chromosome arm level loss events that are common to PC/PGL tumors (e.g. chr1, chr3q, chr17; Fig. 2A) (Castro-Vega et al. 2015b). We previously detected a TP53 mutation in case V-PH-03T by whole exome sequencing (Flynn et al. 2015), and given the known functional role of mutant TP53 in causing genomic instability in cancer (reviewed by Kastenhuber \& Lowe 2017), this may explain the higher number of structural variants observed in this case. No deleterious 
A

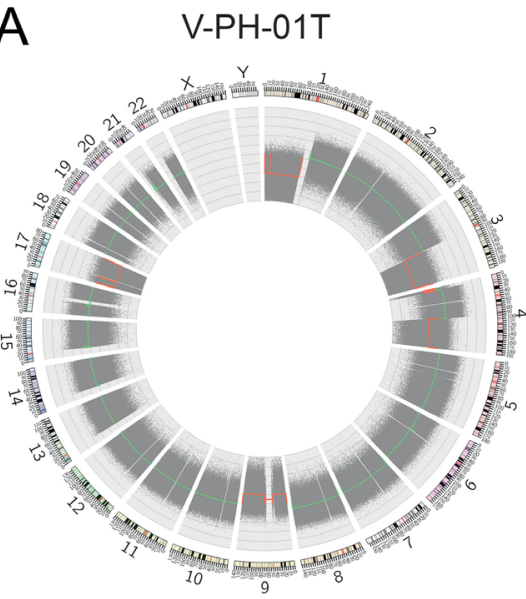

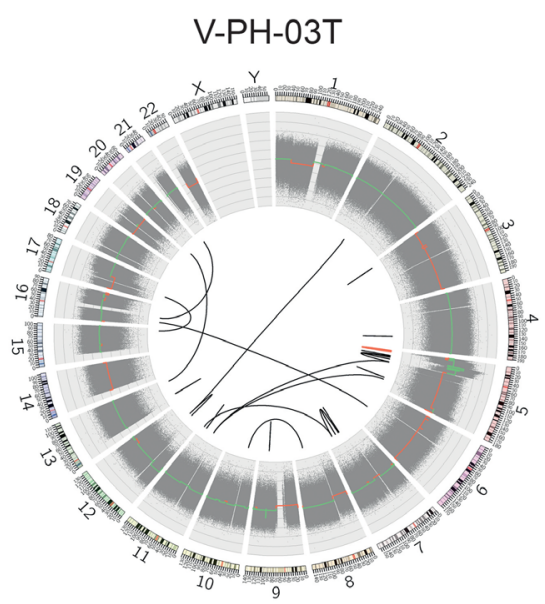

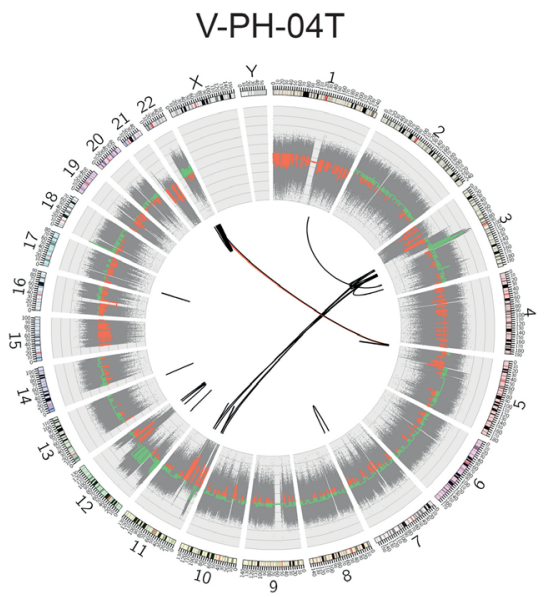

B<smiles>C1CC1</smiles>

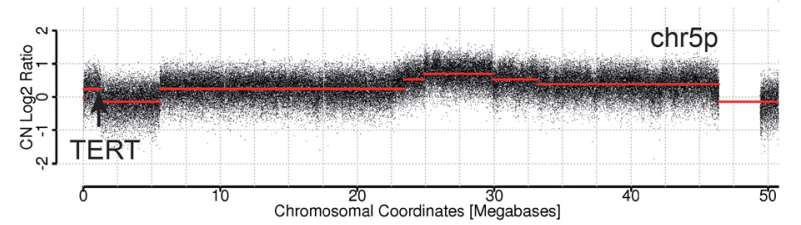

D

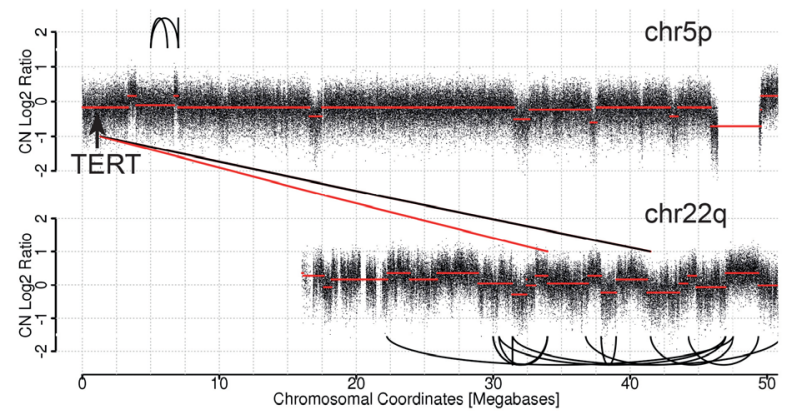

C

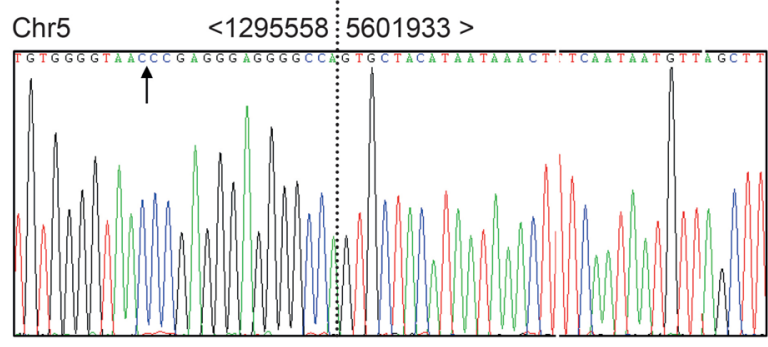

E

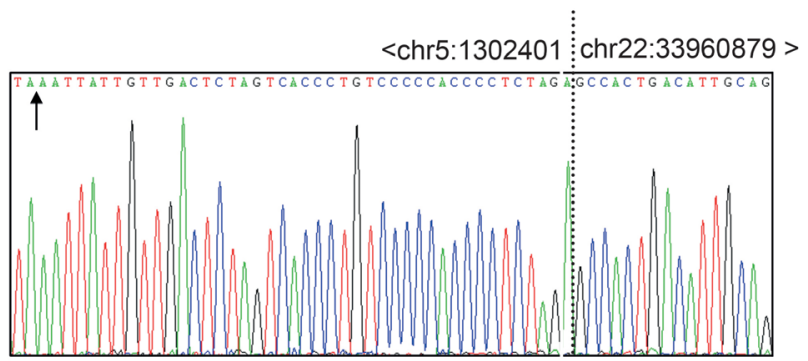

Figure 2

Structural alterations involving the TERT promoter identified in metastatic pheochromocytomas. (A) Circos plots displaying global somatic copy-number and breakpoints detected by WGS analysis across the three TERT overexpressing PC tumors. No somatic breakpoints were detected in V-PH-01T. Chromosome ideograms displayed as outer ring, somatic copy-number changes detected as inner ring. Green line indicates neutral (diploid) or copy-number gain from segmentation, and red line indicates copy-number loss. Inner lines show detected breakpoints. Red lines highlight the validated TERT promoter rearrangements. Data for chrX and chrY not shown. (B) Chromosome level view of somatic copy-number alterations and breakpoints in $\mathrm{V}-\mathrm{PH}-03 \mathrm{~T}$ highlighting an intra-chromosomal deletion detected proximal to TERT as indicated by red line. (C) Sanger sequencing validation of $4.3 \mathrm{Mb}$ deletion upstream of TERT in V-PH-03T. (D) Chromosome level view of somatic copy-number alterations and breakpoints in V-PH-04T involving chr5p and chr22q. Red line indicates TERT proximal breakpoint. (E) Sanger sequencing validation of non-reciprocal translocation (t(5;22)(p15.3;q12.3)) identified in $\mathrm{V}-\mathrm{PH}-04 \mathrm{~T}$. Arrows in panels $\mathrm{C}$ and $\mathrm{E}$ indicates TERT proximal structural breakpoint previously described in aggressive neuroblastoma (Peifer et al. 2015).

somatic mutations in genes associated with genome maintenance could be identified in cases V-PH-01T or V-PH-04T.

Somatic breakpoints were detected in close proximity to TERT in V-PH-03T and V-PH-04T. In V-PH-03T, a $4.3 \mathrm{Mb}$ intra-chromosomal deletion was identified (Fig. 2B and C) corresponding to breakpoints at chr5:1295558 (454 bp upstream of the TERT transcription start site (TSS)) and at chr5:5601933 (a non-coding region of the genome, with the closest gene (LINCO2145) located approximately 
$708 \mathrm{~Kb}$ upstream). The $4.3 \mathrm{Mb}$ deletion in V-PH-03T was further supported by segmental hemizygous loss (as detected by copy-number analysis) and occurred in the context of localized genomic instability on chr5p involving several additional rearrangements and focal amplifications centromeric of TERT.

In V-PH-04T, a non-reciprocal inter-chromosomal translocation $(\mathrm{t}(5 ; 22)(\mathrm{p} 15.3 ; \mathrm{q} 12.3))$ was detected arising from a breakpoint at chr5:1302401 (approximately $7.3 \mathrm{~Kb}$ upstream of the TERT TSS) being juxtaposed to a break at chr22:33960879 ((c.742G, NM_004737) within exon 7 of LARGE1 (a member of the $\mathrm{N}$-acetylglucosaminyltransferase gene family)) (Fig. 2D and E). Again breakpoints on chr5 and chr22 in V-PH-04T arose in the context of marked genomic instability involving localized regions of copynumber loss and gain on both chromosomes.

\section{TERT structural alterations involve enhancer elements}

To interpret the functional effect of TERT rearrangements with respect to cis regulatory elements, we assessed adrenal specific super-enhancer proximity using annotation from dbSUPER (an integrative database of super-enhancers (Khan \& Zhang 2016)) defined by histone H3K27 acetylation in normal adult adrenal tissue (GEO Accession: GSM1013168). The location of super-enhancer regions was further supported by ENCODE data (v3, enabling visualization of enhancer-like regions) representing enrichment peaks for $\mathrm{H} 3 \mathrm{~K} 27$ acetylation and DNase hypersensitivity in fetal adrenal tissue (GEO: GSM1160190) (Rosenbloom et al. 2013). The 4.3 Mb intrachromosomal deletion (chr5:1295558-chr5:5601933) in $\mathrm{V}-\mathrm{PH}-03 \mathrm{~T}$ resulted in the positioning of a super-enhancer (SE_01397) within 1.1 Mb of the TERT TSS (Fig. 3A), while the non-reciprocal translocation $(\mathrm{t}(5 ; 22)(\mathrm{p} 15.3 ; \mathrm{q} 12.3))$ involving juxtaposition of LARGE1 (chr22:33960879) to the TERT promoter (chr5:1302401) in V-PH-04T resulted in the positioning of a super-enhancer (SE_01144) within $1.9 \mathrm{Mb}$ of the TERT TSS (Fig. 3B). In contrast, the closest super-enhancer (SE_01397 (chr5: 6704085-6734741)) within the normal reference genome is positioned $5.4 \mathrm{Mb}$ upstream of the TERT TSS.

Interestingly, additional enhancer-like elements identified in fetal adrenal tissue were positioned approximately $3 \mathrm{~Kb}$ and $18 \mathrm{~Kb}$ upstream of the TERT TSS when assessing the $4.3 \mathrm{Mb}$ deletion (chr5:1295558chr5:5601933) in V-PH-03T (Supplementary Fig. 2A). When assessing the non-reciprocal translocation $(\mathrm{t}(5 ; 22)$ (p15.3;q12.3)) in V-PH-04T, enhancer-like regions in fetal adrenal tissue were observed $577 \mathrm{~Kb}$ and $593 \mathrm{~Kb}$ upstream of the TERT TSS (Supplementary Fig. 2B). In summary, somatic structural alterations where found in two of
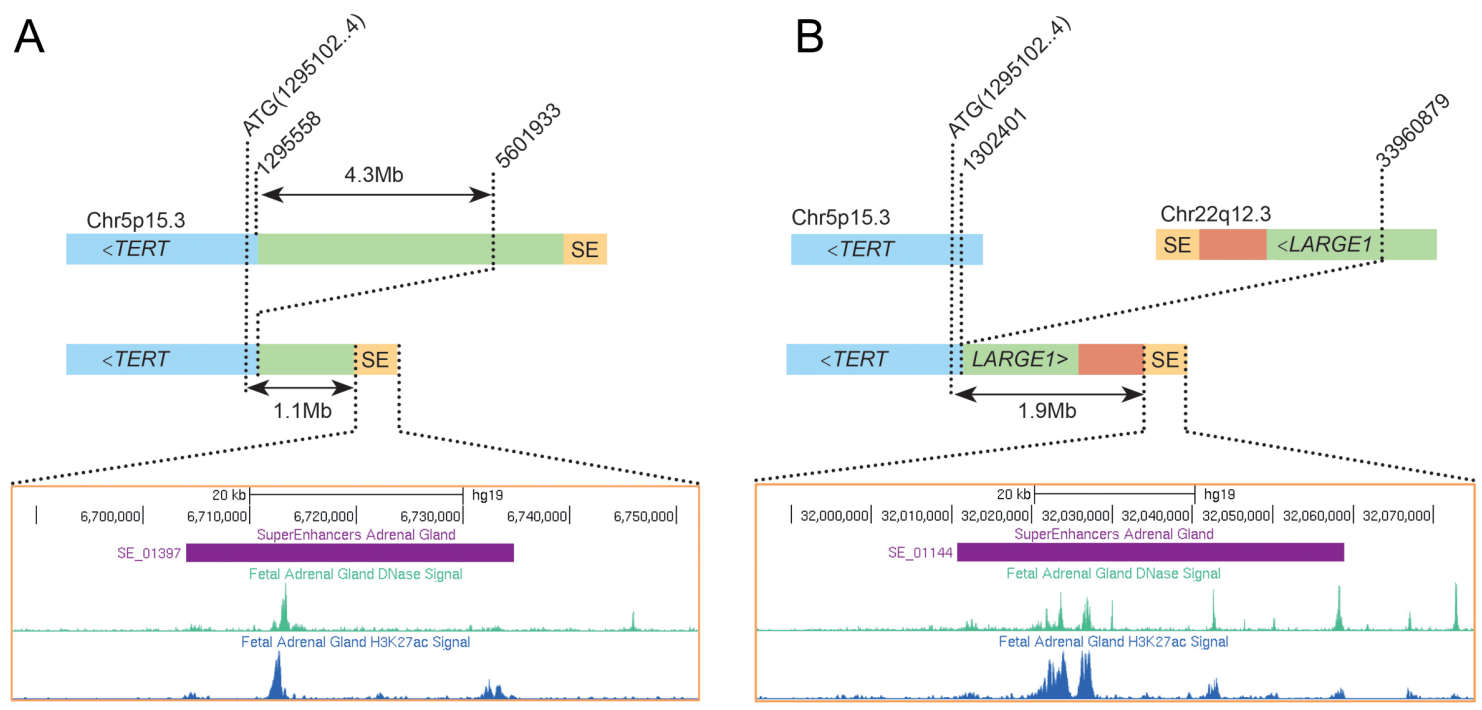

Figure 3

Structural rearrangements juxtapose TERT proximal to active enhancers in metastatic PC. The potential for enhancer-driven activation of TERT was assessed using dbSUPER (an integrative database of super-enhancers; Khan \& Zhang 2016). Candidate super-enhancers are based on H3K27ac signals from normal adult adrenal tissue (GEO accession: GSM1013168). ENCODE data for H3K27ac (blue track) and DNase hypersensitivity (green track) in fetal adrenal tissue supporting super-enhancer features was accessed through UCSC browser (build hg19) (Rosenbloom et al. 2013). (A) The 4.3 Mb somatic deletion observed in V-PH-03T is predicted to result in placement of a super-enhancer 1.1 Mb from the TERT TSS (SE_01397, dbSUPER). (B) The somatic non-reciprocal translocation, $\mathrm{t}(5 ; 22)(\mathrm{p} 15.3 ; \mathrm{q} 12.3)$, identified in $\mathrm{V}-\mathrm{PH}-04 \mathrm{~T}$ is predicted to result in the placement of a super-enhancer $1.9 \mathrm{Mb}$ from the TERT TSS (SE_01144, dbSUPER). 
the three TERT overexpressing PC tumors that resulted in juxtaposition of super-enhancer and enhancer-like elements close to the TERT promoter.

\section{Discussion}

Re-activation of TERT is common to many cancers, and it is mediated by genetic or epigenetic variation at the TERT locus (Barthel et al. 2017). In PC/PGL, TERT expression is typically low to undetectable in non-metastatic cases and increased in a subset of metastatic tumors (Liu et al. 2014). In this study, we examined the mechanism for increased TERT expression in three metastatic PCs, and by using WGS, somatic structural rearrangements involving the TERT promoter in two cases were identified for the first time in this disease; in each case, the structural variants resulted in super-enhancer positioning proximal to the TERT transcription start site (TSS), which are likely responsible for TERT activation.

In our PC/PGL cohort, TERT expression (as assessed by RNA-seq) was detectable in 20 of 39 (51\%) cases, which included five of six metastatic cases; of these, two metastatic PCs had markedly elevated TERT expression and a third metastatic PC showed moderately elevated TERT expression. Our data closely resemble those of Liu and coworkers who found detectable TERT expression by RT-PCR in 18 of 75 (24\%) PC/PGL (Liu et al. 2014). In that study, six $(8 \%)$ tumors (including three metastatic cases) showed markedly elevated TERT expression and 12 (16\%) had moderately elevated expression. Further analysis of TERT mRNA gene expression in 178 primary PC/PGL, assessed by The Cancer Genome Atlas (TCGA), showed 34 (19\%) primary tumors with detectable TERT expression (Fishbein et al. 2017). Importantly, two of the top three TERT-expressing tumors in the TCGA dataset were metastatic (Supplementary Table 4). Taken together, moderate-to-high TERT expression appears in a minority of PC/PGL tumors, but we confirm this group is enriched in tumors with a metastatic phenotype.

None of our three TERT-overexpressing cases were associated with germline $S D H B$ mutations, which are well known to be associated with increased risk of metastasis in PC/PGLs (Benn et al. 2015). Exome sequencing of V-PH-01T and V-PH-04T had identified somatic NF1 (p.Thr467HisfsTer6 (ENSP00000351015.4)) and VHL (p.Ile109Ser (ENSP00000256474.2)) mutations, respectively, while no somatic or germline mutation involving a known PC/PGL gene was detected in V-PH-03T (Flynn et al. 2015). Similarly, of the top five
TERT-expressing tumors in the TCGA dataset, none harbored $S D H B$ mutations, but included one hereditary $V H L$-associated tumor and four tumors of unknown gene driver. Telomere dysfunction through alternate lengthening of telomeres (ALT) has also been associated with metastatic PC/PGLs. Fishbein and coworkers identified somatic ATRX mutations in two SDHB-associated PC/PGLs and in addition, 13 of 103 (12.6\%) of a validation cohort of PC/PGLs, many of which were associated with clinically aggressive behavior (Fishbein et al. 2015). However, in our prior study, only 2 of 39 (5.1\%) PC/PGLs had somatic protein-truncating $A T R X$ mutations, both of which were non-metastatic and neither associated with germline $S D H B$ mutation (Flynn et al. 2015). Owing to the long latency of disease progression in PC/PGL, it is plausible that such non-metastatic cases may still develop metastases in time. We conclude that telomere dysfunction through somatic mutation or structural alterations affecting TERT or ATRX are likely to be predictors of metastatic risk in PC/PGL and can arise independently of $S D H B$ driver mutations.

Given the shared neural crest origin of PC/PGL and neuroblastoma, there is a striking parallel between telomere dysfunction due either to TERT expression or ATRX loss of function in these two diseases (Peifer et al. 2015, Valentijn et al. 2015). Indeed, structural rearrangements involving the upstream region of the TERT promoter were observed in 21\% (Peifer et al. 2015) and 23\% (Valentijn et al. 2015) of neuroblastomas and occurred only in high-risk cases that did not have MYCN amplification or ATRX mutations (Peifer et al. 2015, Valentijn et al. 2015). Interestingly, the TERT breakpoints identified in PC tumors from our study, at chr5:1295558 (V-PH-03T) and chr5:1302401 (V-PH-04T) occur within $15 \mathrm{bp}$ and $44 \mathrm{bp}$, respectively, of breakpoints observed in two neuroblastoma cases from a previous neuroblastoma study (Fig. 2C (NBL54: chr5:1295550) and Fig. 2E (NBL40: chr5:1302358), respectively (Peifer et al. 2015)). These correlative observations in neuroblastoma further validate the likely functional effect of TERT rearrangements in PC/PGL.

Our study emphasizes the value of using WGS to detect TERT rearrangements as a plausible cause of TERT overexpression in metastatic PC/PGL, which would have been missed by studies employing whole exome sequencing (Castro-Vega et al. 2015b, Flynn et al. 2015, Toledo et al. 2016, Fishbein et al. 2017). It was evident that the two TERT-rearranged tumors identified in our study displayed higher degrees of genomic instability, indicating that defects in genome maintenance are important mechanisms for 
perturbation of PC/PGL driver genes. Indeed, we and others have previously identified chromothripsis in PC/PGL tumors and structural rearrangements involving known PC/PGL tumor suppressor genes, such as NF1 (Castro-Vega et al. 2014, Flynn et al. 2015), while the recent TCGA study described putative oncogenic MAML3 and BRAF gene fusions (Fishbein et al. 2017). Ongoing structural instability during PC/PGL evolution has also been described as a characteristic of metastatic PC/PGL (Crona et al. 2015). However, it is apparent that some metastatic PC/PGL can also have relatively stable genomes, as we observed in case V-PH-01T. It is curious that despite the use of WGS and a thorough targeted search for canonical mutations and CpG methylation events in the TERT promoter, we could not detect genetic or epigenetic events explaining TERT overexpression in tumor V-PH-01T. Manual inspection of the WGS data in $\mathrm{V}-\mathrm{PH}-01 \mathrm{~T}$ revealed an uneven and low read coverage in the TERT promoter that precluded variant detection outside of the two known TERT promoter mutation hotspots. Deeper sequencing of the TERT promoter for detection of non-canonical mutations may be warranted to provide a higher sensitivity of detection in future studies. It is also plausible that TERT can be dysregulated by another currently unknown mechanism in some PC/PGL tumors.

In summary, analysis of genomic data from a subset of PC/PGL has identified a role for genomic rearrangements as a cause of TERT overexpression in PC/PGL. Further analysis in larger sets of these tumors will be required to verify the frequency of TERT rearrangements in PC/PGL and to validate their association with aggressive disease. Furthermore, additional analysis is required to verify the canonical and non-canonical functions of TERT in PC/PGL tumors (Martinez \& Blasco 2011). Finally, a number of therapeutic strategies (such as telomerase inhibition, TERT promoter-driven therapy and TERT-directed immunotherapy) are currently under investigation, which may lead to improved outcomes in individuals with TERT-overexpressing metastatic PC/PGL tumors (Xu \& Goldkorn 2016).

Supplementary data

This is linked to the online version of the paper at http://dx.doi.org/10.1530/ ERC-17-0306.

\section{Declaration of interest}

The authors declare that there is no conflict of interest that could be perceived as prejudicing the impartiality of the research reported.

\section{Funding}

This work was supported by grants from the National Health and Medical Research Council (APP1108032 to R.C.B., R.W.T., T.D., D.E.B., A.J.G and APP1053792 to RJH), Hillcrest Foundation (Perpetual Trustees; T.D., D.E.B., B.G.R.), Pheo Para Alliance (T.D., R.C.B., D.E.B., B.G.R.) and the National Breast Cancer Foundation to A.D. The Olivia Newton-John Cancer Research Institute acknowledges the support of the Victorian Government Operational Infrastructure Support Program.

\section{Author contribution statement}

$T D, R C B$ and $R$ W T conceived the study and participated in its design and coordination. T D, A F, K A, R L, J L, D L C, S B, I L M C, S Q W, A T P and $A D$ performed experiments and analyses. A J G performed pathology review. D E B, A H, B G R, R J H and R C B recruited patients and/or curated clinical data. T D, R C B and R W T wrote the manuscript, with critical review provided by $A F, K A, D E B, R L, J L, D L C, A H, S B, I L M C, S Q W, B$ $G R, A T P, A J G, A D, R J H$. All authors have read and approved the final manuscript; take public responsibility for appropriate portions of content and are accountable in relation to the accuracy and integrity of the work.

\section{Acknowledgements}

The authors thank the Kolling Neuroendocrine Tumour Bank for provision of specimens; Dr Martyn Bullock for insights into the TERT promoter and Dr Sarah-Jane Dawson for assistance and contribution of reagents.

\section{References}

Alaei-Mahabadi B, Bhadury J, Karlsson JW, Nilsson JA \& Larsson E 2016 Global analysis of somatic structural genomic alterations and their impact on gene expression in diverse human cancers. PNAS 113 13768-13773. (https://doi.org/10.1073/pnas.1606220113)

Barthel FP, Wei W, Tang M, Martinez-Ledesma E, Hu X, Amin SB, Akdemir KC, Seth S, Song X, Wang Q, et al. 2017 Systematic analysis of telomere length and somatic alterations in 31 cancer types. Nature Genetics 49 349-357. (https://doi.org/10.1038/ng.3781)

Benn DE, Robinson BG \& Clifton-Bligh RJ 201515 YEARS OF PARAGANGLIOMA: Clinical manifestations of paraganglioma syndromes types 1-5. Endocrine-Related Cancer 22 T91-T103. (https://doi.org/10.1530/ERC-15-0268)

Cameron DL, Schroeder J, Penington JS, Do H, Molania R, Dobrovic A, Speed TP \& Papenfuss AT 2017 GRIDSS: sensitive and specific genomic rearrangement detection using positional de Bruijn graph assembly. Genome Research [in press].

Castelo-Branco P, Choufani S, Mack S, Gallagher D, Zhang C, Lipman T, Zhukova N, Walker EJ, Martin D, Merino D, et al. 2013 Methylation of the TERT promoter and risk stratification of childhood brain tumours: an integrative genomic and molecular study. Lancet Oncology 14 534-542. (https://doi.org/10.1016/\$14702045(13)70110-4)

Castro-Vega LJ, Buffet A, De Cubas AA, Cascon A, Menara M, Khalifa E, Amar L, Azriel S, Bourdeau I, Chabre O, et al. 2014 Germline mutations in $\mathrm{FH}$ confer predisposition to malignant pheochromocytomas and paragangliomas. Human Molecular Genetics 23 2440-2446. (https://doi.org/10.1093/hmg/ddt639)

Castro-Vega LJ, Lepoutre-Lussey C, Gimenez-Roqueplo AP \& Favier J $2015 a$ Rethinking pheochromocytomas and paragangliomas from a genomic perspective. Oncogene 35 1080-1089. (https://doi. org/10.1038/onc.2015.172)

Castro-Vega LJ, Letouze E, Burnichon N, Buffet A, Disderot PH, Khalifa E, Loriot C, Elarouci N, Morin A, Menara M, et al. $2015 b$ Multi-omics analysis defines core genomic alterations in 
pheochromocytomas and paragangliomas. Nature Communications 6 6044. (https://doi.org/10.1038/ncomms7044)

Crona J, Backman S, Maharjan R, Mayrhofer M, Stalberg P, Isakson A, Hellman P \& Bjorklund P 2015 Spatio-temporal heterogeneity characterizes the genetic landscape of pheochromocytoma and defines early events in tumourigenesis. Clinical Cancer Research 21 4451-4460 (https://doi.org/10.1158/1078-0432.CCR-14-2854)

Davis CF, Ricketts CJ, Wang M, Yang L, Cherniack AD, Shen H, Buhay C, Kang H, Kim SC, Fahey CC, et al. 2014 The somatic genomic landscape of chromophobe renal cell carcinoma. Cancer Cell $\mathbf{2 6}$ 319-330. (https://doi.org/10.1016/j.ccr.2014.07.014)

Ding Z, Mangino M, Aviv A, Spector T, Durbin R \& Consortium UK 2014 Estimating telomere length from whole genome sequence data. Nucleic Acids Research 42 e75. (https://doi.org/10.1093/nar/gku181)

Fishbein L, Khare S, Wubbenhorst B, DeSloover D, D'Andrea K, Merrill S, Cho NW, Greenberg RA, Else T, Montone K, et al. 2015 Wholeexome sequencing identifies somatic ATRX mutations in pheochromocytomas and paragangliomas. Nature Communications 6 6140. (https://doi.org/10.1038/ncomms7140)

Fishbein L, Leshchiner I, Walter V, Danilova L, Robertson AG, Johnson AR, Lichtenberg TM, Murray BA, Ghayee HK, Else T, et al. 2017 Comprehensive molecular characterization of pheochromocytoma and paraganglioma. Cancer Cell 31 181-193. (https://doi.org/10.1016/j.ccell.2017.01.001)

Flynn A, Benn D, Clifton-Bligh R, Robinson B, Trainer AH, James P, Hogg A, Waldeck K, George J, Li J, et al. 2015 The genomic landscape of phaeochromocytoma. Journal of Pathology 236 78-89. (https://doi.org/10.1002/path.4503)

Horn S, Figl A, Rachakonda PS, Fischer C, Sucker A, Gast A, Kadel S, Moll I, Nagore E, Hemminki K, et al. 2013 TERT promoter mutations in familial and sporadic melanoma. Science 339 959-961. (https://doi.org/10.1126/science.1230062)

Kastenhuber ER \& Lowe SW 2017 Putting p53 in context. Cell 170 1062-1078. (https://doi.org/10.1016/j.cell.2017.08.028)

Khan A \& Zhang X 2016 dbSUPER: a database of super-enhancers in mouse and human genome. Nucleic Acids Research 44 D164-D171. (https://doi.org/10.1093/nar/gkv1002)

Lee S, Borah S \& Bahrami A 2017a Detection of aberrant TERT promoter methylation by combined bisulfite restriction enzyme analysis for cancer diagnosis. Journal of Molecular Diagnostics 19 378-386. (https://doi.org/10.1016/j.jmoldx.2017.01.003)

Lee M, Napier CE, Yang SF, Arthur JW, Reddel RR \& Pickett HA 2017b Comparative analysis of whole genome sequencing-based telomere length measurement techniques. Methods 114 4-15. (https://doi. org/10.1016/j.ymeth.2016.08.008)

Li H 2013 Aligning sequence reads, clone sequences and assembly contigs with BWA-MEM. arXiv:1303.3997v2 [q-bio.GN]. (available at: https://arxiv.org/abs/1303.3997)

Liu T, Brown TC, Juhlin CC, Andreasson A, Wang N, Backdahl M, Healy JM, Prasad ML, Korah R, Carling T, et al. 2014 The activating TERT promoter mutation C228T is recurrent in subsets of adrenal tumors. Endocrine-Related Cancer 21 427-434. (https://doi. org/10.1530/ERC-14-0016)

Martinez P \& Blasco MA 2011 Telomeric and extra-telomeric roles for telomerase and the telomere-binding proteins. Nature Reviews Cancer 11 161-176. (https://doi.org/10.1038/nrc3025)

Papathomas TG, Oudijk L, Zwarthoff EC, Post E, Duijkers FA, van Noesel MM, Hofland LJ, Pollard PJ, Maher ER, Restuccia DF, et al. 2014 Telomerase reverse transcriptase promoter mutations in tumors originating from the adrenal gland and extra-adrenal paraganglia. Endocrine-Related Cancer 21 653-661. (https://doi.org/10.1530/ERC13-0429)

Peifer M, Hertwig F, Roels F, Dreidax D, Gartlgruber M, Menon R, Kramer A, Roncaioli JL, Sand F, Heuckmann JM, et al. 2015 Telomerase activation by genomic rearrangements in high-risk neuroblastoma. Nature 526 700-704. (https://doi.org/10.1038/ nature14980)

Ramlee MK, Wang J, Toh WX \& Li S 2016 Transcription regulation of the Human Telomerase Reverse Transcriptase (hTERT) gene. Genes 7 50. (https://doi.org/10.3390/genes7080050)

Robinson JT, Thorvaldsdottir H, Winckler W, Guttman M, Lander ES, Getz G \& Mesirov JP 2011 Integrative genomics viewer. Nature Biotechnology 29 24-26. (https://doi.org/10.1038/nbt.1754)

Rosenbloom KR, Sloan CA, Malladi VS, Dreszer TR, Learned K, Kirkup VM, Wong MC, Maddren M, Fang R, Heitner SG, et al. 2013 ENCODE data in the UCSC genome browser: year 5 update. Nucleic Acids Research 41 D56-D63. (https://doi.org/10.1093/nar/gks1172)

Shen R \& Seshan VE 2016 FACETS: allele-specific copy number and clonal heterogeneity analysis tool for high-throughput DNA sequencing. Nucleic Acids Research 44 e131. (https://doi.org/10.1093/nar/gkw520)

Tischler AS, de Krijger R, Gill AJ, Kawashima A, Komminoth P, Papathomas T, Thompson LDR, Tissier F, Williams MD \& Young WF 2017 Tumors of the adrenal medulla and extra-adrenal paraganglia. In WHO Classification of Tumours of Endocrine Organs. 4th Edition. Eds Lloyd RV, Osamura RY, Klöppel G \& Rosai J. Lyon, France: IARC Press.

Toledo RA, Qin Y, Cheng ZM, Gao Q, Iwata S, Silva GM, Prasad ML, Ocal IT, Rao S, Aronin N, et al. 2016 Recurrent mutations of chromatin-remodeling genes and kinase receptors in pheochromocytomas and paragangliomas. Clinical Cancer Research 22 2301-2310. (https://doi.org/10.1158/1078-0432.CCR-15-1841)

Valentijn LJ, Koster J, Zwijnenburg DA, Hasselt NE, van Sluis P, Volckmann R, van Noesel MM, George RE, Tytgat GA, Molenaar JJ, et al. 2015 TERT rearrangements are frequent in neuroblastoma and identify aggressive tumors. Nature Genetics 47 1411-1414. (https://doi.org/10.1038/ng.3438)

Wong SQ, Waldeck K, Vergara IA, Schroder J, Madore J, Wilmott JS, Colebatch AJ, De Paoli-Iseppi R, Li J, Lupat R, et al. 2015 UV-associated mutations underlie the etiology of MCV-negative merkel cell carcinomas. Cancer Research 75 5228-5234. (https://doi. org/10.1158/0008-5472.CAN-15-1877)

$\mathrm{Xu}$ Y \& Goldkorn A 2016 Telomere and telomerase therapeutics in cancer. Genes 7 22. (https://doi.org/10.3390/genes7120125)

Received in final form 30 September 2017

Accepted 3 October 2017

Accepted preprint published online 3 October 2017 